\title{
Fast noninertial shifts of attention
}

\author{
DOV SAGI and BELA JULESZ \\ AT \& T Bell Laboratories, Murray Hill, NJ 07974, USA
}

Received 8 February 1985; accepted in revised form 3 July 1985

\begin{abstract}
It was suggested that some discrimination tasks (e.g. discrimination between the letters $\mathrm{T}$ and $\mathrm{L}$ ) require serial search by scrutinizing each letter (target) with a small aperture of focal attention. Here we examine the effect of intertarget distance on discrimination performance, using two targets. We find reduction in performance at short distances, in agreement with masking studies, but constant performance independent of distance outside this masking region. This constant performance is still lower than expected from masking effects and might reflect attentive processes. Sequential presentation of the targets with delays up to $30-40 \mathrm{~ms}$, while reducing available processing time, does not cause reduction in performance, thus supporting the suggestion that discrimination of the two targets is a serial process. The independence of performance on distance suggests fast noninertial shifts of attention.
\end{abstract}

\section{INTRODUCTION}

It was suggested recently that the visual system can find differences in density of features in parallel, while detecting differences in positional relationship between features (Bergen and Julesz, 1983) or conjunctions of features (Treisman and Gelade, 1980 ) calls for serial scan by focal attention. This serial scrutiny is much faster than eye movements and is required even for the discrimination of well-resolvable targets. For example, detecting a line element surrounded by many other line elements of different orientation can be done in parallel, but knowing the orientation of the detected line element requires additional serial processing, even for the largest orientation differences of $90^{\circ}$ (Sagi and Julesz, 1983). So knowing what the target is requires scrutiny by focal attention even though the target has adequate visual acuity. Thus we might have a first stage parallel preattentive visual system, with the primary task of detecting density differences of local features, followed by a serial system inspecting these local differences. This serial search is probably directed by the preattentive system (Julesz, 1981, Julesz and Bergen, 1983).

Here we examine dynamic properties of attention shifts, in particular we are interested in the time course of the shift. We study the effect of varying the distance between two targets on the ability of observers to compare them (e.g. to tell whether they are the same or different). This experimental paradigm differs from the classical masking paradigm by the requirement of discrimination between the two targets. Masking experiments where an observer has to identify a letter in the presence of a masking letter show short-range interactions (Bouma, 1970); reduction in performance occurs when the masking letter is close to the target letter, within a distance of half the eccentricity of the target. Outside this range the mask has no effect and the target can be identified with the same accuracy with or without the mask. Assuming that masking effect is symmetrical in space we might conclude that if the masker letter does not mask the target letter, the inverse relationship holds, the masker is not masked by the target. From that reasonable assumption we can conclude that when two letters are outside 
each other's masking range they can be identified with the same accuracy as each of them separately and thus if one letter can be identified without error we should expect error-free identification and comparison of the two letters. Any reduction in performance in the two letter discrimination task can be attributed to interactions due to the discrimination task.

Here we show that when a single letter can be identified perfectly (as a $T$ or $L$ ) two such letters cannot be compared without errors even outside the classical masking range. This reduction in performance is independent of the inter-letter distance as long as this distance is greater than half the presentation eccentricity. This effect implies some kind of interference at the discrimination level. This is in support of the suggestion that the discrimination task might require serial processing while multiple detections can be done in parallel (Sagi and Julesz, 1983, 1985).

We used a discrimination task that involves focal attention shifts. The task of detecting the relative position of two orthogonal line segments, can be best visualized as discrimination between the letters T and L. Bergen and Julesz (1983) using a masking paradigm, where duration of visual persistence is limited and processing time can be controlled, showed that detecting the presence of one $\mathrm{L}$ (or $\mathrm{T}$ ) among a variable number of Ts (or Ls) is affected by the number of targets presented. They suggested that this result indicates that serial processing is involved in the detection task. Here we add support to their suggestion by showing that the visual system cannot discriminate between two widely separated letters even if each of them can be identified separately. More than that we show that the failure is due to the discrimination task and not due to the presence of the second letter. Contrary to classical masking effects the strength of this effect is independent of distance. This independence of distance holds also under sequential presentation (by presenting the second target after a delay) thus forcing the task to be serial.

The two targets to be discriminated were presented at constant eccentricity, with their position randomized on the perimeter of a circle having its center at the fixation point. Each target position was randomized independently, thus randomizing also the distance between them and keeping distance uncertainty. We also varied the circle diameter, but scaled the target size in accordance with the 'Aubert-Foerster law' (see von Helmholtz, 1925), doubling the target size when doubling eccentricity.

\section{METHOD}

We chose a discrimination task between two letters, T and L, each randomly oriented. The observers were presented with a pair of letters ( $T \& T, T \& L, L \& T, L \& L$ ), as targets, and had to decide whether the two targets were the same or not. Error rates were measured as the average error rates on the two alternatives. Experiments were divided into blocks of 50 trials each, that were repeated two to four times with the same experimental conditions; data points presented in the results section represent averages from 100-200 trials. Different tasks, SOAs and delays were presented in different blocks of trials, inter-target distances were always intermixed randomly within each block of trials.

The two targets were presented at the same distance from the fixation point (see Fig. 1 for demonstration) where the target size was scaled according to this distance. In most of the experiments the targets, of $0.9^{\circ}$ size, were presented at $4^{\circ}$ distance from the fixation point, thus enabling us to measure the effect of inter-target distances up to $8^{\circ}$. We performed also experiments at a distance of $12^{\circ}$ from the fixation point with a target 
$\perp$
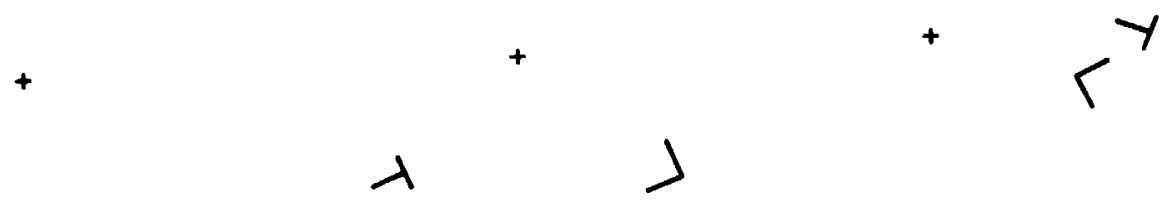

$<$

Figure 1. Three possible arrangements of the target pairs. Note that for all targets distance from fixation point is approximately equal. The left and right configurations are of the largest and shortest intertarget distances, respectively.

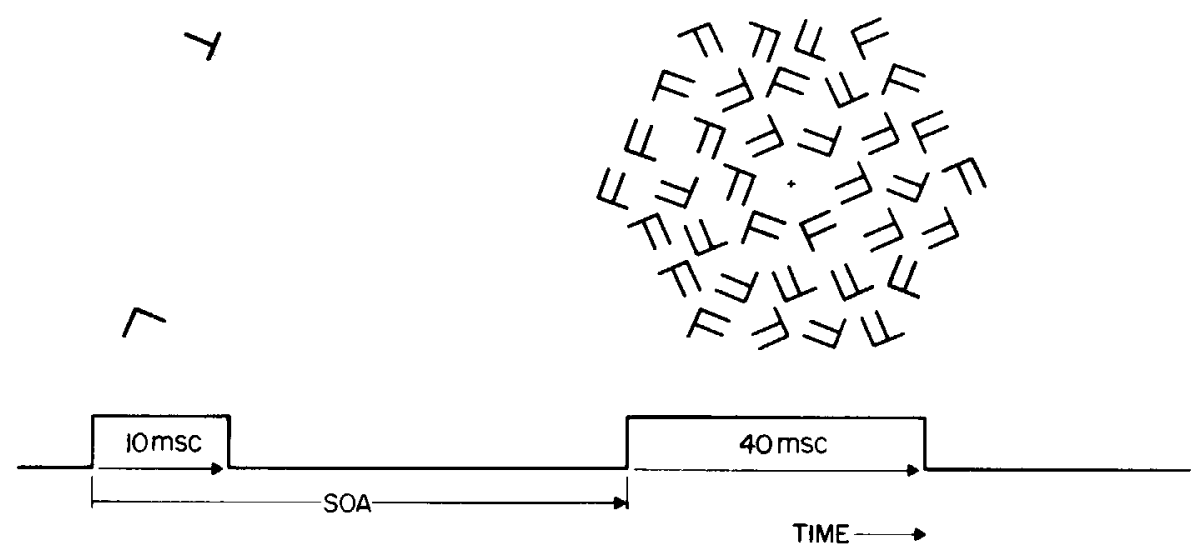

Figure 2. Temporal presentation of the stimuli. The targets were presented for $10 \mathrm{~ms}$ and the mask for $40 \mathrm{~ms}$. The mask had three shells, where the third one had 18 elements corresponding to the 18 possible locations of the targets.

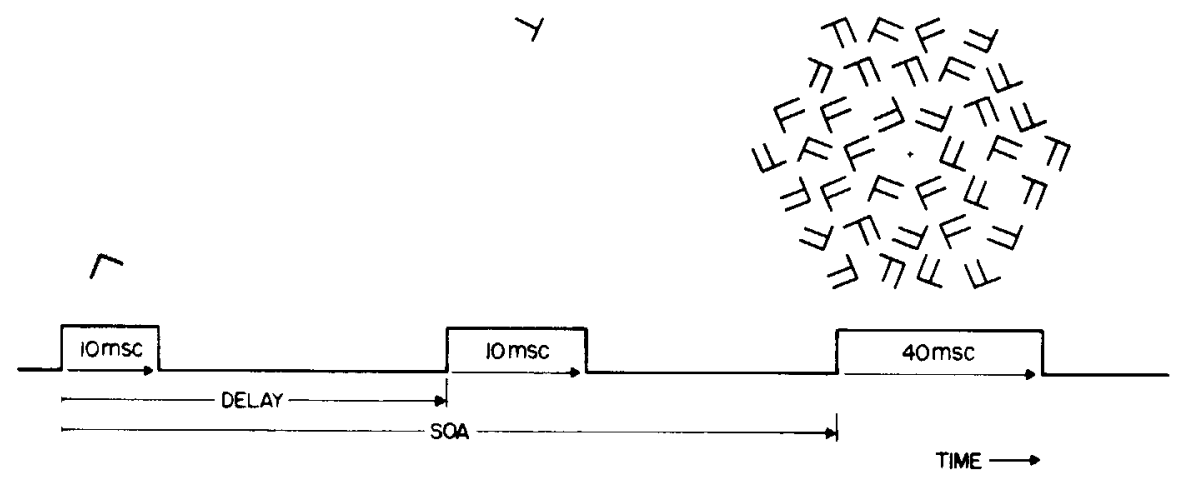

Figure 3. Temporal presentation in experiment where one target was delayed. 
size of $2.7^{\circ}$, allowing inter-letter distances up to $24^{\circ}$ (all spatial dimensions scaled up by a factor of three). For the $4^{\circ}$ and $12^{\circ}$ eccentricity, respectively, all the different intertarget distances were intermixed in each block of trials (50 trials), thus yielding distance uncertainty for the observers.

The targets, white on a black background, were presented briefly for $10 \mathrm{~ms}$ (preventing a second fixation during the exposure) and were followed by a patterned mask ( $40 \mathrm{~ms}$ duration) as shown in Fig. 2 . Each mask element, of the 36 elements is the union of the two letters $\mathrm{T}$ and $\mathrm{L}$. The time interval between the targets and the mask (stimulus onset asynchrony $=\mathrm{SOA}$ ) was usually kept constant, to allow comfortable performance of $80-90 \%$ correct response. The two targets were presented simultaneously (Fig. 2) or sequentially (Fig. 3), where one of the targets was delayed relative to the other. The stimuli were computer generated and displayed on the face of a cathode ray tube HP $1310 \mathrm{~A} X-Y$ display. Viewing distance was $1 \mathrm{~m}$.

The observers serving in the experiments were all well practiced, and except for one (D.S.) were not aware of the purpose of the experiments.

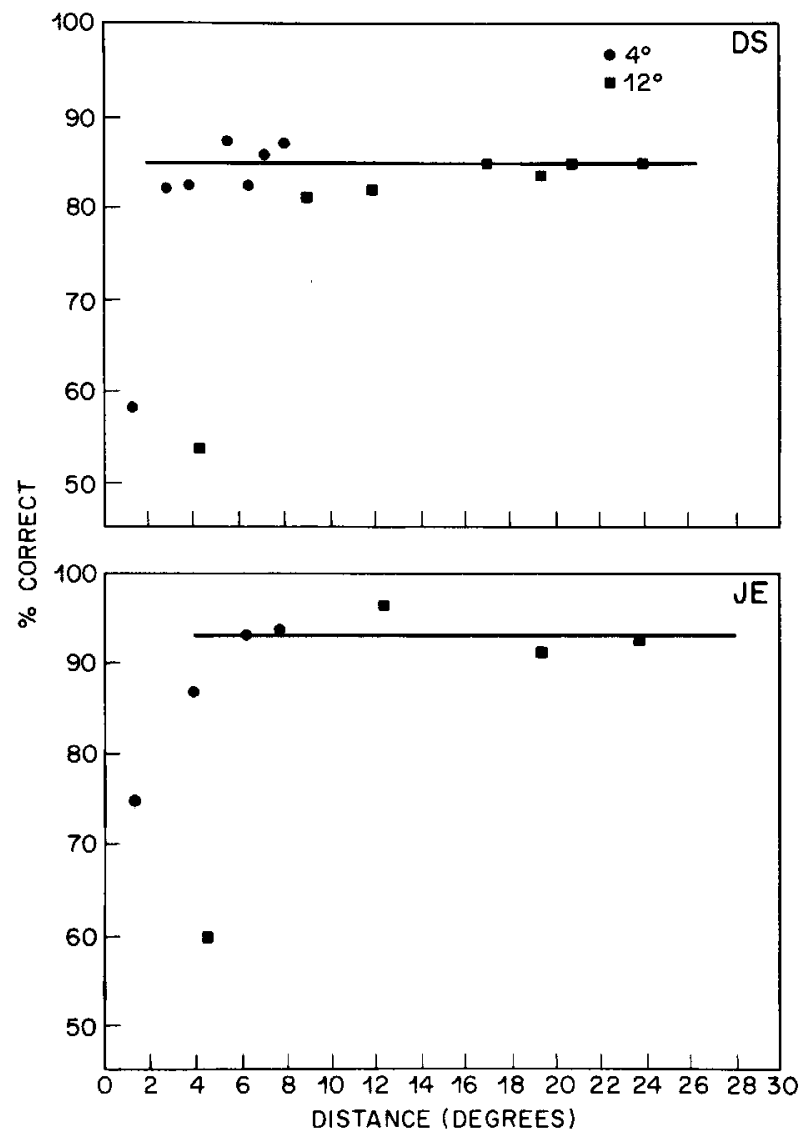

Figure 4. The effect of distance on percentage correct discrimination of $T$ and $L$, at SOA of $90 \mathrm{~ms}$. Targets were presented either at eccentricity of $4^{\circ}(0)$ or $12^{\circ}(\boldsymbol{D})$, the target size was scaled with distance. Performance is approximately constant, except for the shortest distance on both eccentricities (Observers D.S. and J.E.) 


\section{RESULTS}

The effect of spatial separation on two targets discrimination

The main results of this study are shown in Fig. 4, where the percentage of correct discrimination is plotted against distance. The shorter distances up to $8^{\circ}$ were measured at $4^{\circ}$ eccentricity, the longer distances, between $8^{\circ}$ and $24^{\circ}$, were measured at $12^{\circ}$ eccentricity. The size of targets presented at $12^{\circ}$ eccentricity was scaled up three times relative to the target presented at $4^{\circ}$ eccentricity. Different distances were intermixed in the same blocks of trials but not different eccentricities. As Fig. 4 shows, performance is constant over most of the range of distances measured; there is a reduction in performance when the distance is smaller than $2^{\circ}$ for the small eccentricity or $6^{\circ}$ for the larger eccentricity. Note that performance is invariant under linear spatial scaling, there is practically no difference between performance at the two eccentricities used when the target size was scaled accordingly. That is in agreement with the 'Aubert-Foerster law' (von Helmholtz, 1925).

\section{Two targets discrimination versus single target identification}

The results depicted in Fig. 4 show reduction in performance when the two targets are close to each other. That might be the result of lateral masking. The range of the effect is about half the targets' eccentricity, a range known as a masking range (Bouma, 1970). However, contrary to masking the effect, here the flat region in Fig. 4 shows reduced performance compared to the discriminability of the two targets when presented alone. In this particular case the two observers could discriminate, under the same conditions, between $\mathrm{T}$ and $\mathrm{L}$ presented alone, one by one, without any error (out of 200 trials). Psychometric functions for discrimination of one or two targets are shown in Fig. 5.

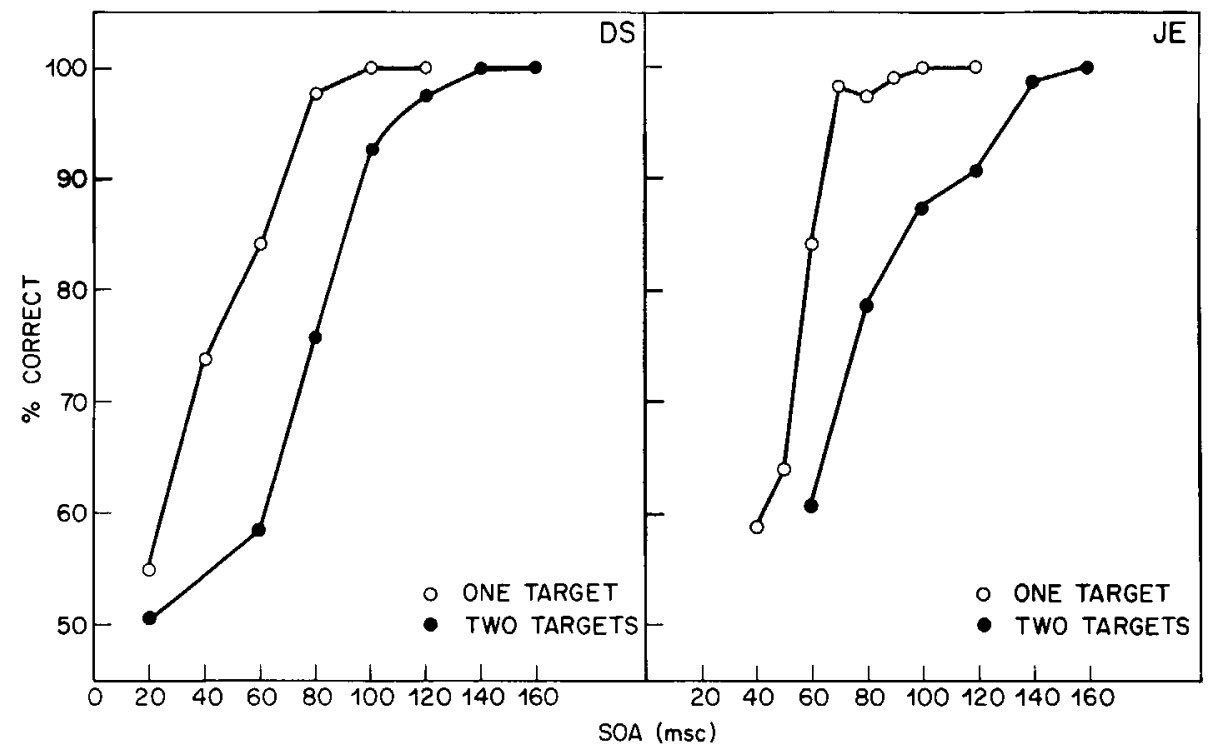

Figure 5. Dependence of correct discrimination on SOA, for identification of one target $(O)$ and discrimination of two targets (O). Discrimination of two targets takes more time than one target identification. Note that at SOA of $100 \mathrm{~ms}$ one target can be identified without error, but two targets can be discriminated correctly on only $90 \%$ of the trials. (Observers J.E. and D.S.) 
In one case the observers were presented with only one target ( $T$ or $L$ ) and were asked to identify it; in the other case they were presented with two targets (with an inter-target distance greater than $3^{\circ}$ ) and were asked to report whether they were the same or not. It is clear from Fig. 5 that processing two targets takes more time, furthermore there is a considerable rate of error in the two-target discrimination task even when one of them alone can be identified without error. Assuming that two-target discrimination requires identification of each of the targets (an assumption which is not necessarily true, the availability of the two targets on the same trial might make the discrimination task easier) then no discrimination errors are expected at SOAs where there are no identification errors. From Fig. 5 we can see that the processing of the additional letter adds about $40 \mathrm{~ms}$ to the processing time, this addition in processing time suggests serial processing of the targets (Treisman and Gelade, 1980; Bergen and Julesz, 1983).

The next point we verified is the possibility that the reduced discrimination performance is because of the presence of a second target (which in our case might be the same as the other target). That was done in an experiment where we presented always two targets at $4^{\circ}$ eccentricity, separated by different distances (see Fig. 2), but one of them had a fixed position during each block of trials (that is $4^{\circ}$ above the fixation point or to the right of the fixation point). The observers performed two tasks, one to identify the non-fixed target as $\mathrm{T}$ or $\mathrm{L}$, the other to compare the two letters. The first task is similar to tasks performed in masking experiments, the second is a replication of the discrimination experiment we described above (with the difference of having one target with a fixed position). Here we used the same stimuli for the two tasks, the only difference being the requirement to process the second letter in order to compare it to the first. The results are depicted in Fig. 6. It is clear that processing the second letter dramatically reduces performance. This reduction in performance might be attributed to the discrimination process itself.

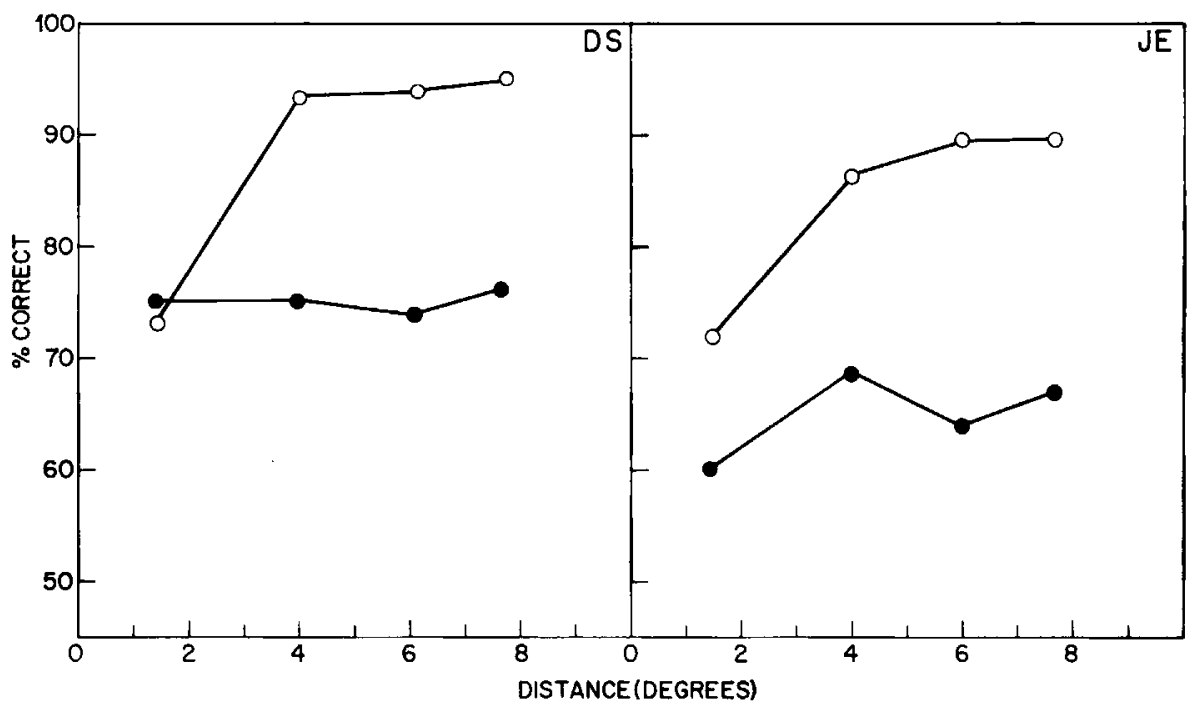

Figure 6. Dependence of identification and discrimination on distance. Stimuli consisted of two targets; one of them having fixed position and the other appeared at different positions at the same eccentricity $\left(4^{\circ}\right)$. The observers had to identify the nonfixed target (open symbols) or to compare the two targets (solid symbols). SOA was kept constant, $60 \mathrm{~ms}$ for observer J.E. and $75 \mathrm{~ms}$ for D.S. 


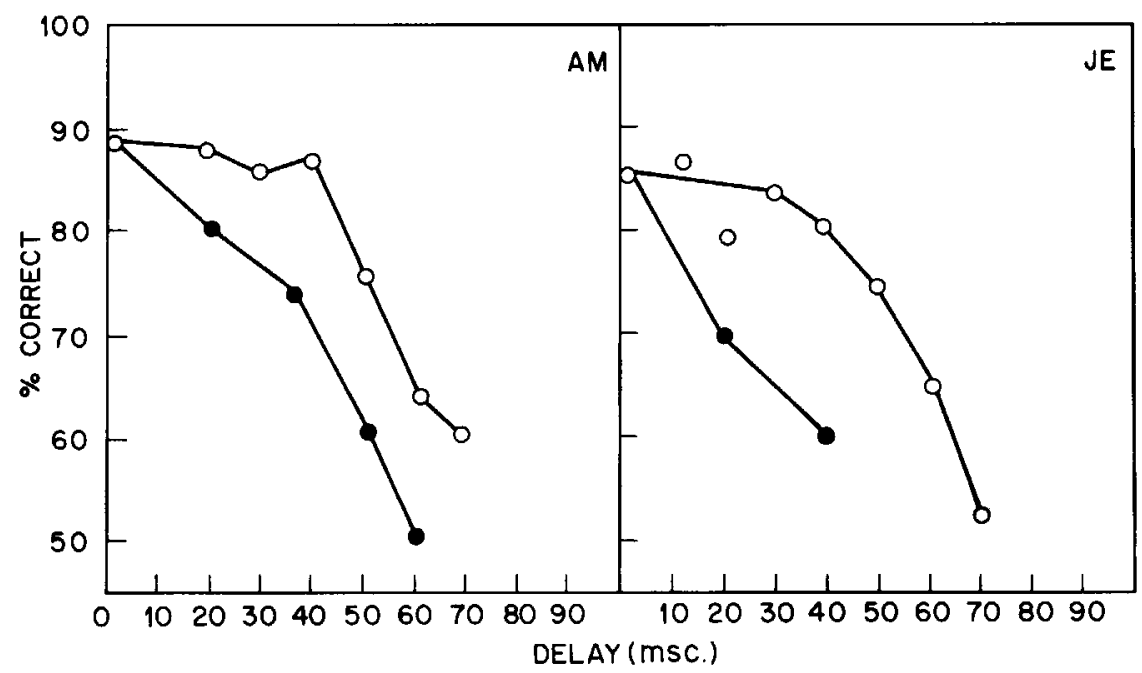

Figure 7. Effect of delaying one target on discrimination (curve marked by $O$ ), compared to delaying two targets ( ). SOA was kept constant for both observer (A.M. and J.E.) at $100 \mathrm{~ms}$. Note that one target can be delayed by up to $40 \mathrm{~ms}$ without affecting performance in a significant way.

Sequential versus simultaneous presentation

The results shown in Fig. 4-6 are of experiments in which the two targets were presented simultaneously. In addition we performed some experiments with sequential presentation of the targets. First we examined the effect on performance of delaying one of the targets while keeping SOA (Stimulus Onset Asynchrony between the first target and the mask) constant. We assumed that if the targets were to be processed serially, performance would not be degraded by sequential presentation. However, if the targets were to be processed in parallel, sequential presentation would degrade performance. The effect of delaying one target is shown in Fig. 7 for two observers. It is shown that

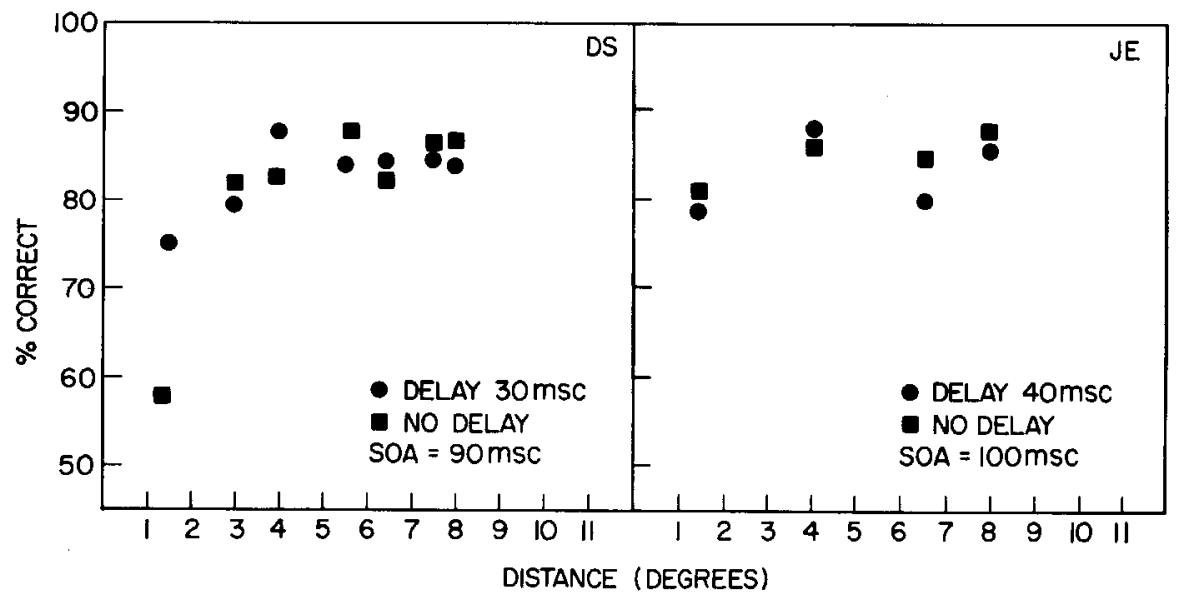

Figure 8. Effect of distance on performance when one target was delayed $(\odot)$ and when presentation was simultaneous ( $\square$ ). Delay was $30 \mathrm{~ms}$ (observer D.S., SOA $=90 \mathrm{~ms}$ ) and $40 \mathrm{~ms}$ (observer, J.E., SOA $=100 \mathrm{~ms}$ ). Note the absence of distance effect. 
one target (out of two) can be delayed by $30-40 \mathrm{~ms}$ without affecting performance. This number agrees with the processing time of $30-50 \mathrm{~ms}$ per target ( $\mathrm{T} \& \mathrm{~L}$ ) found by Bergen and Julesz (1983). On the other hand delaying two targets, which is equivalent to shortening the SOA, has an immediate effect on performance.

Figure 8 presents result of experiments where the distance between the targets was varied as in Fig. 4, but here the targets were presented also sequentially. The result is similar to that presented in Fig. 4; there is no notable effect of distance on performance either in the sequential presentation case or in the simultaneous presentation case.

\section{DISCUSSION}

The results of our experiments show that there is no distance effect in discrimination tasks requiring inspection by shifting focal attention. We found that inspection duration was constant for separation distances up to $24^{\circ}$ of visual angle, and independent of eccentricity as long as scaled targets were presented. The only deviation from this constant performance was found when the two targets were adjacent, thus interfering with each other by lateral masking.

We found that even outside this classical range of masking there is some interference; observers, while able to identify each of the two targets separately, could not avoid errors in the two targets discrimination task. Thus the visual system is limited in its ability to perform multiple identifications in parallel. This is in agreement with the finding of Bergen and Julesz (1983) where they suggested that this task involves serial processing, at the rate of $30-50 \mathrm{~ms}$ per target. That suggestion is further supported by our finding that one of the two targets in the two targets discrimination task could be delayed by up to $30-40 \mathrm{~ms}$ without affecting performance. What is surprising here is that two distant targets (up to $24^{\circ}$ separation) interfere with each other in discrimination task, only when both have to be attended, however attention can be shifted between the two targets with the same efficiency regardless of the distance between them. Thus the time it takes to shift attention from one target to the other is independent of distance.

Previous studies showed effect of distance on attention shift durations (Shulman et al., 1979; Tsal, 1983) but recently Remington and Pierce (1984) have found no effect of distance on duration of attention shifts. These experiments usually involve measuring reaction time for detecting targets at expected locations compared to unexpected locations at different distances from fixation point. A basic assumption, critical to the interpretation of the data from these experiments, is that differences in performance between the expected and the unexpected locations conditions reflect attentional mechanism. These experiments raise also some technical problems, the intermixing of distance and eccentricity complicates the interpretation of the data since the effectiveness of the stimuli is different at different eccentricities. One way to compensate for peripheral sensitivity reduction is using targets that are easier to discriminate (Tsal, 1982), however that introduces another complication of having to compare different kinds of targets, thus the improvement in discrimination might reflect decreased similarity between targets instead of compensating for low resolution (see the distinction between 'data-limit' and 'resource-limit' by Norman and Bobrow, 1975). The experimental design described in this paper bypassed the above problems by separating the distance parameter from the eccentricity parameter and by choosing a discrimination task that involves serial processing.

To summarize, we did not find distance effect in two targets discrimination tasks. The finding that duration of focal attention shifts for discrimination is independent of 
distance might not be so surprising if the underlying process were parallel; however, as we showed here the process seems to be serial. Discrimination tasks might bring the visual system to its limit of parallel processing. Nevertheless, even in discrimination tasks the locations of the targets might be detected in parallel, thus enabling fast inertiafree shifts of attention between locations. Understanding the mechanism of assigning positions to luminance gradients, or feature gradients in general, and of directing attention to these positions is a strategic problem of brain research.

\section{REFERENCES}

Bergen, J. R. and Julesz, B. (1983). Parallel verus serial processing in rapid pattern discrimination. Nature 303, 696-698.

Bouma, H. (1970). Interaction effects in parafoveal letter recognition. Nature 226, 177-178.

von Helmholtz, H. (1925). Treatise on Physiological Optics, Vol. 2. J. P. C. Southall (Ed.). Optical Society of America (pp. 34-35).

Julesz, B. (1981). Textons, the elements of texture perception and their interactions. Nature 290, 91-97.

Julesz, B. and Bergen, J. R. (1983). Textons, the fundamental elements in preattentive vision and perception of textures. Bell Sys. Tech. J. 62, 1619-1645.

Norman, D. L. and Bobrow, D. G.(1975). On data-limited and resource-limited processes Cognitive Psychol. 7, 44-64.

Remington, R. and Pierce, L. (1984). Moving attention: Evidence for time-invariant shifts of visual selective attention. Percept. Psychophys. 35, 393-399.

Sagi, D. and Julesz, B. (1983). Rapid counting versus pattern discrimination in preattentive vision. J. Opt. Soc. Am. 73, 1924.

Sagi, D. and Julesz, B. (1985). 'Where' and 'What' in vision. Science 228, 1217-1219.

Shulman, G. L., Remington, R. W. and McLean, J. P. (1979). Moving attention through visual space. J. Exp. Psychol. 5, 522-526.

Treisman, A. and Gelade, G. (1980). A feature integration theory of attention. Cognitive Psychol. 12, 97-136.

Tsal, Y. (1983). Movements of attention across the visual field. J. Exp. Psychol. 9, 523-530. 